\title{
Lentivirus-mediated overexpression of miR-124 suppresses growth and invasion by targeting JAG1 and EZH2 in gastric cancer
}

\author{
YANGYANG PAN $^{1}$, AIHUA WU ${ }^{2}$, FANFAN XU ${ }^{1}$, CHAO CHEN $^{1}$, LEI JIANG ${ }^{2}$ and RONG JIN ${ }^{1,3}$ \\ ${ }^{1}$ Department of Gastroenterology; ${ }^{2}$ Central Laboratory; ${ }^{3}$ Department of Epidemiology, \\ The First Affiliated Hospital of Wenzhou Medical University, Wenzhou, Zhejiang 325000, P.R. China
}

Received July 16, 2016; Accepted January 26, 2018

DOI: $10.3892 / 01.2018 .8194$

\begin{abstract}
MicroRNA-124 (miR-124) expression is downregulated and has a tumor suppressor role in various types of cancer. The present study revealed that genes encoding miR-124 were frequently methylated and the expression of miR-124 was downregulated in gastric cancer tissues. Stable expression of miR-124 using a lentiviral vector inhibited gastric cancer cell growth, migration and invasion in vitro. In addition, overexpression of miR-124 suppressed gastric cancer cell xenograft growth in nude mice. The expression of the Notch ligand Jagged1 (JAG1) and enhancer of zeste homolog 2 (EZH2) was downregulated upon miR-124 overexpression, and silencing of JAG1 or EZH2 by RNA interference also suppressed gastric cancer cell growth, migration and invasion. Furthermore, expression of fibronectin and vimentin, not able elements of the epithelial-mesenchymal transition, were suppressed by overexpression of miR-124 or inhibition of JAG1 or EZH2 expressions in GC. Together, these results indicated that miR-124 suppressed gastric cancer progression, partly through inhibiting JAG1 and EZH2. Thus, lentivirus-mediated overexpression of miR-124 may be a potential therapeutic strategy against gastric cancer.
\end{abstract}

\section{Introduction}

Despite decreasing rates of morbidity, gastric cancer (GC) remains a high-incidence neoplastic disease that is particularly prevalent in Eastern Asia (1); it is the third-leading cause of

Correspondence to: Dr Rong Jin, Department of Epidemiology, The First Affiliated Hospital of Wenzhou Medical University, 1 South Baixiang Street, Wenzhou, Zhejiang 325000, P.R. China

E-mail: jinrongjrjr@163.com

Dr Lei Jiang, Central Laboratory, The First Affiliated Hospital of Wenzhou Medical University, 1 South Baixiang Street, Wenzhou, Zhejiang 325000, P.R. China

E-mail: jiangleistone79@163.com

Key words: microRNA-124, lentivirus, Jagged1, enhancer of zeste homolog 2 , gastric cancer cancer-associated mortality in the world (2). GC rates in men are approximately twice as high as in women (1). To date, although patients with early $\mathrm{GC}$ can receive effective treatment and achieve a clinical cure, GC patients with moderate or advanced GC lack an effective form of therapy.

MicroRNAs (miRNAs/miRs) are small, noncoding RNAs 22 nucleotides in length that have notable functions in development, cell differentiation and regulation of cell cycle and apoptosis (3). miRNAs regulate gene expression generally by inhibiting translation or degrading mRNA transcripts (4). miRNA expression is deregulated in cancer via a variety of mechanisms such as amplification, deletion, mutation, and epigenetic silencing (3).

miRNA-124 is abundantly and specifically expressed in the brain, but is expressed at a low level in other tissues (5); it has a critical role in central nervous system development and function, as well as the progression of various diseases in brain (5). Recently, miR-124 has been found down-regulated in multiple tumor types, including cancer (6), prostate (7), lung (8) and colorectal cancer (9). It also been found that miR-124 could inhibit the proliferation of GC cells proliferation by targeting Rho-associated protein kinase 1, enhancer of zeste homolog 2 (EZH2) and sphingosine kinase 1 (SPHK1) (10-12). A previous study demonstrated that overexpression of miR-124 by transfection with miR-124 mimics could inhibit GC cell growth, migration and invasion, and induce cell cycle arrest by directly targeting the Notch ligand Jagged1 (JAG1) in vitro (13). Furthermore, Notch activation by the ectopic expression of intracellular domain of Notch1 (NICD) negatively regulates miR-124 expression and promotes cell growth, migration and invasion in GC cells (13). A recent study revealed that miR-124 was downregulated in GC tissues and was associated with lymph node metastasis and tumor stages (14).

The results of the present study demonstrated that miR-124 genes were methylated and the expression of miR-124 was downregulated in GC specimens. Lentivirus-mediated overexpression of miR-124 inhibited GC cell growth, migration and invasion, and suppressed of GC cell xenografts growth in a nude mouse model. In addition, overexpression of miR-124 suppressed JAG1 and EZH2 protein expression in GC cells. Silencing of JAG1 or EZH2 by RNA interference inhibited GC cell growth, migration and invasion. The expression of fibronectin and vimentin proteins was downregulated by miR-124 overexpression or silencing of JAG1 or EZH2 in GC cells. 
These data provided additional evidence of a pivotal role for miR-124 in GC, and indicated that overexpression of miR-124 using lentiviruses may be a feasible approach to treatment of GC.

\section{Materials and methods}

Clinical specimens and cell culture. Gastric specimens were collected from $51 \mathrm{GC}$ patients that had received surgery in the First Affiliated Hospital of Wenzhou Medical University (Wenzhou, Zhejiang, China) between January 2014 and December 2015. These GC cases included 40 male and 11 female patients with a mean age of 65 years (range 37-86 years). All patients had not received radiotherapy or chemotherapy prior to surgery. Tissue samples were immediately snap-frozen in liquid nitrogen after resection and stored at $-80^{\circ} \mathrm{C}$ until subsequent analyses. The tissues were verified by a trained pathologist. Normal gastric samples were taken at a distance of at least $5 \mathrm{~cm}$ from the tumor. The collection and analysis of clinical specimens were approved by the Institutional Review Board of the First Affiliated Hospital of Wenzhou Medical University. Written informed consent was obtained from the patients prior to participation.

GC SGC-7901 and AGS cell lines, and 293T cells were purchased from the Type Culture Collection of the Chinese Academy of Sciences (Shanghai, China). SGC-7901 and AGS cells were cultured in RPMI-1640 medium (Invitrogen; Thermo Fisher Scientific, Inc., Waltham, MA, USA), 293T was cultured in Dulbecco's Modified Eagle's Medium (DMEM) (Invitrogen; Thermo Fisher Scientific, Inc.), and each medium were supplemented with $10 \%$ fetal bovine serum (FBS) (Invitrogen; Thermo Fisher Scientific, Inc.) and $100 \mathrm{U} / \mathrm{ml}$ penicillin/streptomycin (Invitrogen; Thermo Fisher Scientific, Inc.). All cells were maintained in a humidified incubator at $37^{\circ} \mathrm{C}$ with $5 \% \mathrm{CO}_{2}$.

Methylation analysis. Genomic DNA was extracted from tissue samples using a DNeasy Blood \& Tissue kit (Qiagen, Inc., Valencia, CA, USA), according to the manufacturer's protocol. An EpiTect bisulfite reagent kit (Qiagen, Inc.) was used to transform 120-200 ng of DNA before it was subjected to polymerase chain reaction (PCR) amplification of the specific promoter region using a primer set (Table I) designed to amplify methylated and unmethylated sequences of the miR-124 genes. This reaction was performed by 2xTaq PCR Master Mix (Tiangen Biotech Co., Ltd., Beijing, China). The thermocycling conditions were as follows: $94^{\circ} \mathrm{C}$ for $5 \mathrm{~min}$, 30 cycles of $94^{\circ} \mathrm{C}$ for $30 \mathrm{sec}, 60^{\circ} \mathrm{C}$ for $30 \mathrm{sec}$, and $72^{\circ} \mathrm{C}$ for $30 \mathrm{sec}$, followed by a final extension at $72^{\circ} \mathrm{C}$ for $10 \mathrm{~min}$. The results were analyzed by $1.5 \%$ agarose gel electrophoresis and ethidium bromide was used to visualize DNA.

Lentiviral vector construction, production and transduction. To construct lentiviral vectors expressing miR-124, miR-124 precursors and their native context sequences (upstream and downstream flanking genomic sequences) were amplified by PCR from 293T cell genomic DNA. The PCR products were digested with SalI/EcoRI restriction enzymes and inserted into corresponding sites of human U6 promoter-containing pBluescript SK (+) plasmid (Stratagene; Aglient Technologies,
Inc., Santa Clara, CA, USA) (15). Next the obtained constructs were digested with $C a l \mathrm{I} / E c o \mathrm{RI}$ and cloned the U6-miRNA cassettes into a previously described lentiviral vector (provided by Dr Chen Yangchao, Chinese University of Hong Kong) (15). Lentivirus carrying green fluorescent protein (GFP) (15) were used as the control. Lentivirus carrying short hairpin RNA (shRNA) targeting firefly luciferase (shLuc, 5'-TGCGCTGCT GGTGCCAACCCTATTCT-3') or EZH2 (shEZH2, 5'-GAC TCTGAATGCAGTTGCTTCAGTACCC-3') was constructed as previously described (16). Cells were transduced with the lentiviruses using polybrene $(8 \mu \mathrm{g} / \mathrm{ml}$; Sigma-Aldrich; Merck $\mathrm{KGaA}$, Darmstadt, Germany).

siRNA transfection. siRNAs for JAG1 (target sequence, 5'-CCU GUAACAUAGCCCGAAA-3') (17) and negative control (NC) siRNA (target sequence, 5'-UUCUCCGAACGUGUCACG U-3') were purchased from GenePharma (GenePharma Co., Ltd., Shanghai, China). Cells were transfected with $50 \mathrm{nM}$ siRNA using Lipofectamine 3000 (Invitrogen; Thermo Fisher Scientific, Inc.) according to the manuscript introduction. Subsequent experimentations were performed $48 \mathrm{~h}$ after transfection.

Reverse transcription-quantitative PCR (RT-qPCR). Total RNA was extracted from tissue samples or cells using TRIzol (Thermo Fisher Scientific Inc., Waltham, MA, USA) according to the manufacturer's instructions. The miR-124 RT reaction was performed with specific RT primer using Revert Aid First Strand cDNA Synthesis kit (Thermo Fisher Scientific Inc.). The expression levels of miR-124, JAG1 and EZH2 were evaluated by qPCR using SYBRGreen PCR Master Mix (Takara Biotechnology Co., Ltd., Dalian, China) and an ABI7500 Real-time PCR system (Applied Biosystems; Thermo Fisher Scientific, Inc.). The thermocycling conditions were as follows: Initial denaturation $95^{\circ} \mathrm{C}$ for $30 \mathrm{sec}$, followed by 40 cycles: $95^{\circ} \mathrm{C}$ for $5 \mathrm{sec}$ and $60^{\circ} \mathrm{C}$ for $34 \mathrm{sec}$. The U6 small nuclear RNA was used for normalization of the mRNA expression level of miR-124. GAPDH, an endogenous housekeeping gene, was used for normalization of the mRNA expression levels of JAG1 and EZH2. The specific RT primers and PCR primers are shown in Table II. The relative difference in expression of studied genes between normal and GC samples was represented by $-\Delta \mathrm{Cq}$. To determine over-expression of miR-124 in GC cells transduced with Lenti-miR-124 relative to control, the $2^{-\Delta \Delta \mathrm{Cq}}$ method was used to calculate fold changes values (18).

Cell proliferation assay. Cell proliferation was determined using the Cell Counting Kit-8 (CCK-8) assay (Dojindo Molecular Technologies, Inc., Kumamoto, Japan). Briefly, 3,000 cells (100 $\mu \mathrm{l} /$ well) were seeded into 96 -well plates and cultured at $37^{\circ} \mathrm{C}$. At $1,2,3$ and 4 days following cell seeding, $10 \mu \mathrm{l}$ CCK-8 solution was added to each well, cells were incubated for another $4 \mathrm{~h}$ at $37^{\circ} \mathrm{C}$. After incubation, a microplate reader was used to measure the corresponding absorbance at $450 \mathrm{~nm}$. Each condition was determined in quintuplicate, and all experiments were repeated three times.

Western blot analysis. Tissue samples or cells were lysed by Radioimmunoprecipitation Assay (RIPA) buffer (Thermo Fisher Scientific, Inc.) supplemented with Protease Inhibitor 
Table I. Primers for methylation-specific polymerase chain reaction.

\begin{tabular}{|c|c|c|c|}
\hline Gene name & Methylation status & Direction & Sequence, 5'-3' \\
\hline \multirow[t]{4}{*}{ miR-124-1 } & M & Forward & AAAGAGTTTTTGGAAGACGTC \\
\hline & & Reverse & AATAAAAAACGACGCGTATA \\
\hline & $\mathrm{N}$ & Forward & AATAAAGAGTTTTTGGAAGATGTT \\
\hline & & Reverse & AAAAAAATAAAAAACAACACATATAC \\
\hline \multirow[t]{4}{*}{ miR-124-2 } & M & Forward & GGGTAATTAATTTGGATTTACGTC \\
\hline & & Reverse & ACCGCTATTAATTAATCTATTCCG \\
\hline & $\mathrm{N}$ & Forward & GGGGTAATTAATTTGGATTTATGTT \\
\hline & & Reverse & AAAACCACTATTAATTAATCTATTCCA \\
\hline \multirow[t]{4}{*}{ miR-124-3 } & $\mathrm{M}$ & Forward & GCGAGGATTTTACGTAAGTTC \\
\hline & & Reverse & CCGCGTACCTTAATTATATAA \\
\hline & $\mathrm{N}$ & Forward & GGGTGAGGATTTTATGTAAGTTT \\
\hline & & Reverse & TTCACCACATACCTTAATTATATAAAC \\
\hline
\end{tabular}

miR-124, microRNA-124; M, methylated; N, non-methylated.

Table II. Primers for RT-quantitative polymerase chain reaction.

\begin{tabular}{|c|c|c|}
\hline Gene name & Direction & Sequence, 5'-3' \\
\hline \multirow[t]{5}{*}{ miR-124 } & RT & GTCGTATCCAGTGCAGGGTC \\
\hline & & CGAGGTATTCGCACTGGATA \\
\hline & & CGACGGCATTCA \\
\hline & Forward & $\begin{array}{l}\text { ATGGTTGGTTGGTAAGGCAC } \\
\text { GCGG }\end{array}$ \\
\hline & Reverse & GCAGGGTCCGAGGTATTC \\
\hline \multirow[t]{3}{*}{ U6 } & RT & AACGCTTCACGAATTTGCGT \\
\hline & Forward & CTCGCTTCGGCAGCACA \\
\hline & Reverse & AACGCTTCACGAATTTGCGT \\
\hline \multirow[t]{2}{*}{ JAG1 } & Forward & GGGGCAACACCTTCAАССТC \\
\hline & Reverse & CCACGCCTCCACAAGCAAC \\
\hline \multirow[t]{2}{*}{$\mathrm{EZH} 2$} & Forward & $\begin{array}{l}\text { CAAAGAGAAAAACCGACT } \\
\text { GCG }\end{array}$ \\
\hline & Reverse & GGCGACGAAGGCTTTGC \\
\hline \multirow[t]{2}{*}{ GAPDH } & Forward & TCCCATCACCATCTTCCAGG \\
\hline & Reverse & GATGACCCTTTTGGCTCCC \\
\hline
\end{tabular}

RT, reverse transcription; JAG1, Jagged1; EZH2, enhancer of zeste homolog 2 .

(Thermo Fisher Scientific, Inc.). The protein concentration was quantified using BCA protein assay kit (Shanghai Qcbio Technologies Co., Ltd., Shanghai, China) according to the manufacturer's protocol. The proteins (40 $\mu \mathrm{g}$ per lane) were separated by $10 \%$ SDS-PAGE. Electrophoresed proteins were transferred to a polyvinylidene fluoride membrane and blocked for $2 \mathrm{~h}$ with $5 \%$ skimmed milk at room temperature, and then incubated with rabbit anti-GAPDH (cat. no. 2118; dilution, 1:1,000; Cell Signaling Technology, Europe, B.V., Leiden, The Netherlands), rabbit anti-JAG1 (cat. no. SC8303; dilution,
1:300; Santa Cruz Biotechnology, Dallas, TX, USA,), rabbit anti-EZH2 (dilution, 1:1,000; Abcam, Cambridge, MA, USA), mouse anti-vimentin (cat. no. 550513; dilution, 1:1,000; BD Biosciences, San Jose, CA, USA), and mouse anti-fibronectin (cat. no. SC18825; dilution, 1:1,000; Santa Cruz Biotechnology, Santa Cruz, CA, USA) primary antibodies at $4^{\circ} \mathrm{C}$ overnight. The next day, the membranes were washed using Tris-buffered saline with 1\% Tween-20 (TBST), and incubated with horseradish peroxidase-labeled secondary anti-mouse (cat. no. 31430; dilution, 1:2,000; Thermo Fisher Scientific, Inc. Waltham, MA, USA) or anti-rabbit IgG antibody (cat. no. 31460; dilution, 1:2,000; Thermo Fisher Scientific, Inc.) at room temperature for $1 \mathrm{~h}$. After washing with TBST, all blots were visualized using enhanced chemiluminescence substrate kit (Amersham, GE Healthcare, Chicago, IL, USA). GAPDH was used as a loading control. The western blot figures were acquired using ChemiDoc $^{\text {TM }}$ XRS+ Imaging Systems (BioRad Laboratories, Inc., Hercules, CA, USA) and densitometry was analyzed using Image Lab 4.1 software (BioRad Laboratories, Inc.).

Cell migration and invasion assays. Cell migration and invasion assays were performed using a Transwell chamber (Corning Incorporated, Corning, NY, USA). Cells $\left(5 \times 10^{5} / 200 \mu \mathrm{l}\right)$ incubated in serum-free RPMI-1640 medium were seeded in the upper chamber; the lower chamber contained complete RPMI-1640 medium supplemented with 10\% FBS. For invasion assays, the upper chambers were pre-coated with Matrigel. After incubation at $37^{\circ} \mathrm{C}$ for $16 \mathrm{~h}$ (migration) or $48 \mathrm{~h}$ (invasion), migratory and invasive cells on the bottom surface were fixed with $4 \%$ paraformaldehyde in room temperature for $30 \mathrm{~min}$ and stained with $0.1 \%$ crystal violet solution, while the cells on the upper membrane were removed. Five fields from each membrane were counted using a x20 objective of light microscope (CKX41; Inverted Microscope, Olympus Corporation, Tokyo, Japan).

Tumor xenografts growth in nude mice. All animal studies complied with current ethical considerations and were 


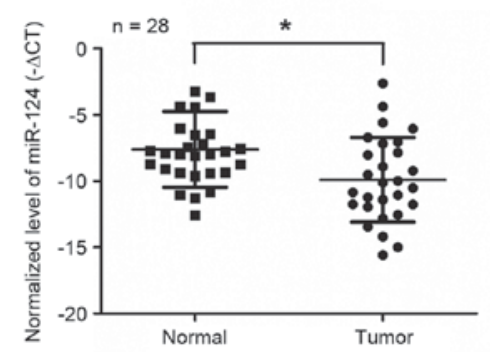

$\mathrm{C}$

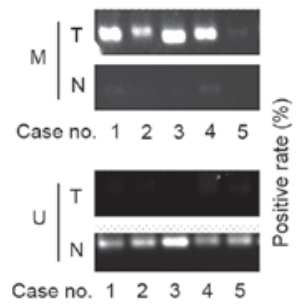

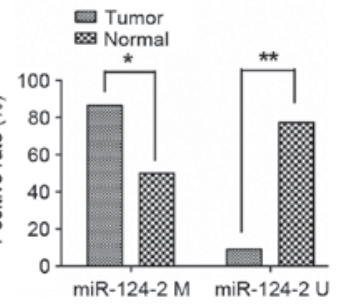

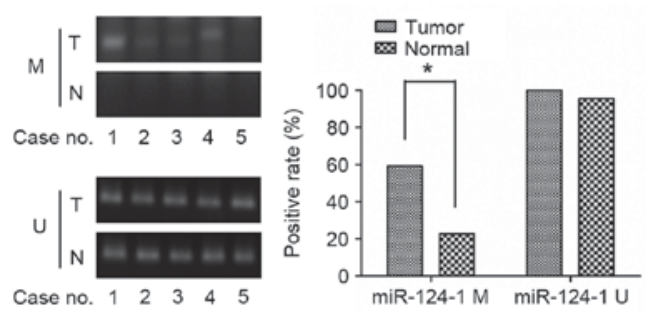

D
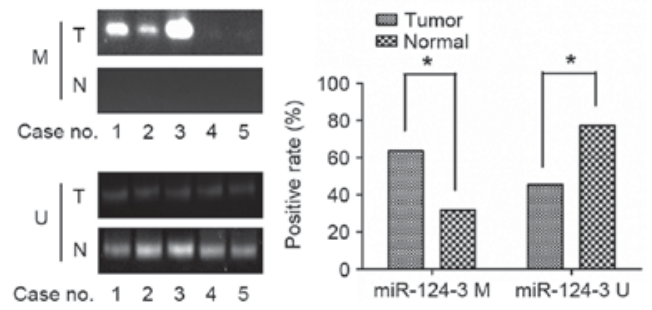

Figure 1. miR-124 genes were frequently methylated and the expression of miR-124 was downregulated in GC tissues. (A) The relative expression levels of miR-124 were detected by reverse transcription-quantitative PCR in 28 cases of GC tissues and matched normal gastric tissues; (B-D) Comparison of miR-124 genes promoter methylation states between GC and matched normal gastric tissues. Methylation of the three miR-124 family genes (miR-124-1, miR-124-2, and miR-124-3) was detected by methylation-specific PCR. miR-124, microRNA-124; M, methylated; U, unmethylated; GC, gastric cancer; miR-124, microRNA-124; T, tumor; $\mathrm{N}$, node; $\mathrm{PCR}$, polymerase chain reaction. ${ }^{*} \mathrm{P}<0.05,{ }^{* *} \mathrm{P}<0.01$.

approved by the Laboratory Animal Ethics Committee of Wenzhou Medical University. A total of $12 \mathrm{BALB} / \mathrm{c}$ nude mice (male, 4-6 weeks old, 18-20 g) were obtained from the Shanghai LAC Laboratory Animal Co. Ltd. (Shanghai, China) and housed in a specific-pathogen-free grade animal center in a $12 \mathrm{~h}$ light/dark cycle under controlled temperature $\left(22-25^{\circ} \mathrm{C}\right)$ and given free access to food and water. Mice were subcutaneously injected with $2.5 \times 10^{6}$ SGC7901 cells in the right side of the back; tumor sizes were then recorded at the indicated times. The humane endpoints were defined as a tumor size exceeding $2,000 \mathrm{~mm}^{3}$ or $>10 \%$ body weight loss. Euthanasia was performed at the endpoint and tumor nodules were dissected.

Statistical analysis. All experiments were performed in triplicate, and data are expressed as the mean \pm standard deviation. Statistical analysis was performed using SPSS 13.0 (SPSS, Inc., Chicago, IL, USA) or GraphPad Prism 5 (GraphPad Software, Inc., La Jolla, CA, USA). Statistically significant differences were calculated using independent samples t-test, or one-way ANOVA followed by post-hoc Tukey's analysis of variance. $\mathrm{P}<0.05$ was considered to indicate a statistically significant difference.

\section{Results}

Expression and methylation analyses of miR-124 in human GC tissues. The expression level of miR-124 in 28 paired GC tissues and adjacent normal tissue were evaluated by RT-qPCR. As Fig. 1 depicts, the miR-124 levels in GC tissues were significantly lower when compared with the normal gastric tissues. miR-124 is present in three genomic loci (miR-124-1 (8p23.1), miR-124-2 (8q12.3) and miR-124-3 (20q13.33)) (19), and miR-124 has been reported to be inactivated by methylation in several tumor types, including hepatocellular carcinoma (20). Therefore, the DNA methylation status of the three CPG sites was detected in 20 paired GC tissues and adjacent normal tissue using methylation-specific PCR. The methylation levels of miR-124-1 (Fig. 1B), miR-124-2 (Fig. 1C) and miR-124-3 (Fig. 1D) were much higher in GC tissues than in normal gastric tissues. These results indicated that miR-124 was hypermethylated and therefore its expression was downregulated in GC.

Lentivirus-mediated overexpression of miR-124 inhibited GC cell growth, migration and invasion. GC cell lines SGC-7901 and AGS cells were transfected with the lentiviral vector expressing miR-124. Overexpression of miR-124 in GC cells was confirmed by RT-qPCR (Fig. 2A and B). To investigate the role of miR-124 in GC cell growth, CCK8 assays were performed. As depicted in Fig. 2A and B, overexpression of miR-124 inhibited SGC-7901 and AGS cell growth.

The effects of miR-124 on migration and invasion of GC cells were evaluated using Transwell migration and invasion assays. As Fig. 2C and D depicts, compared with the empty-vector groups, those transfected with miR-124 significantly inhibited the migratory and invasive abilities of SGC-7901 and AGS cells ( $\mathrm{P}<0.001)$. Ectopic stable expression of miR-124 in SGC-7901 and AGS cells reduced the number of migrated cells by 53.4 and $16.4 \%$ compared with GC cells expressing GFP, respectively, and in the invasion assay by 43.6 and $47.3 \%$, respectively.

To confirm the effect of miR-124 on GC cell growth in vivo, xenograft models were generated by implanting SGC-7901 cells transfected with lentivirus stably overexpressing miR-124 or a control lentiviral vector into nude mice. The results revealed that overexpression of miR-124 led a marked decrease in the rate of subcutaneous xenograft tumor growth (Fig. 2E-G). Together, these findings confirmed that miR-124 inhibits miR-124 GC cell growth, migration and invasion. 

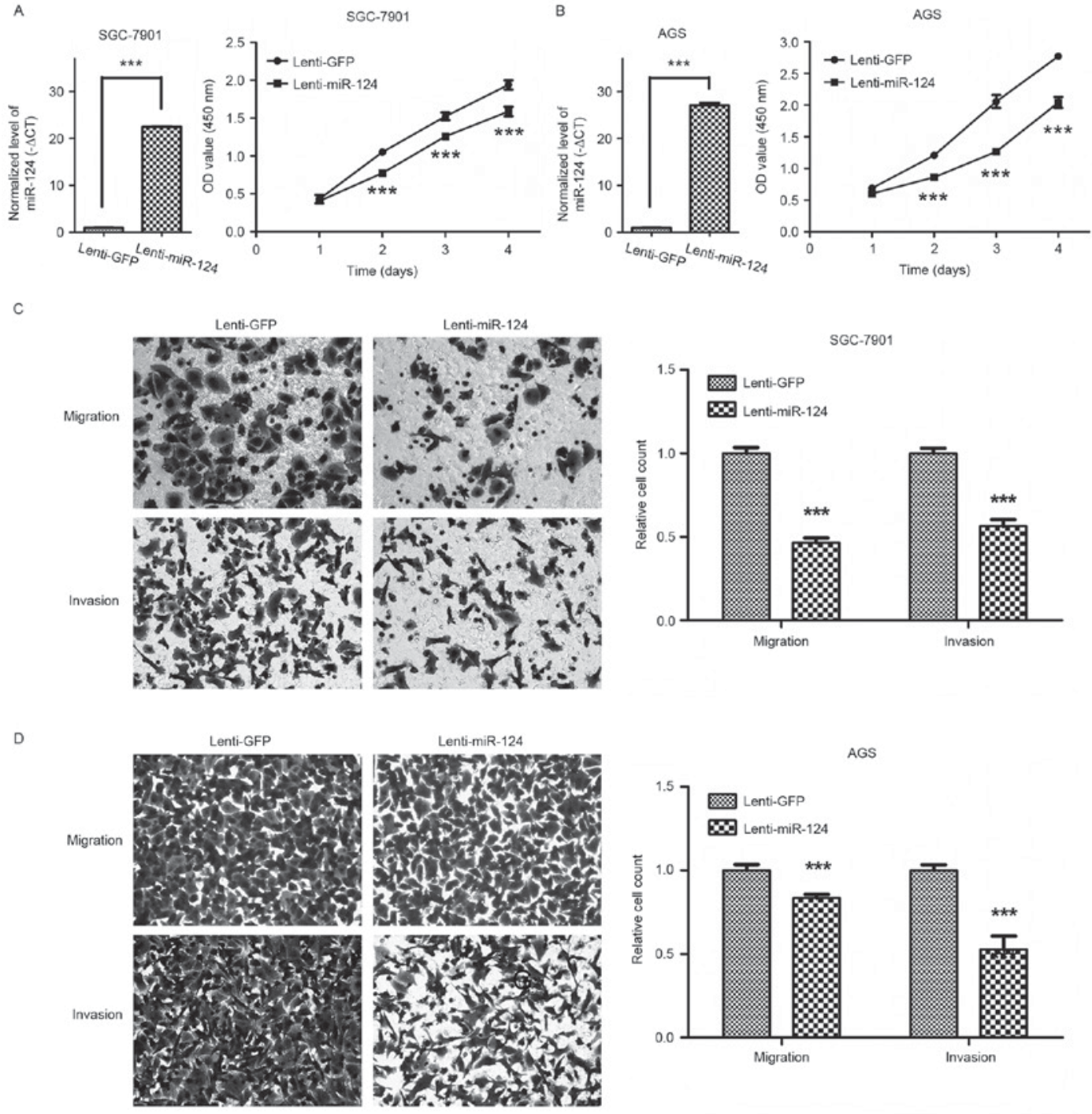

$\mathrm{F}$
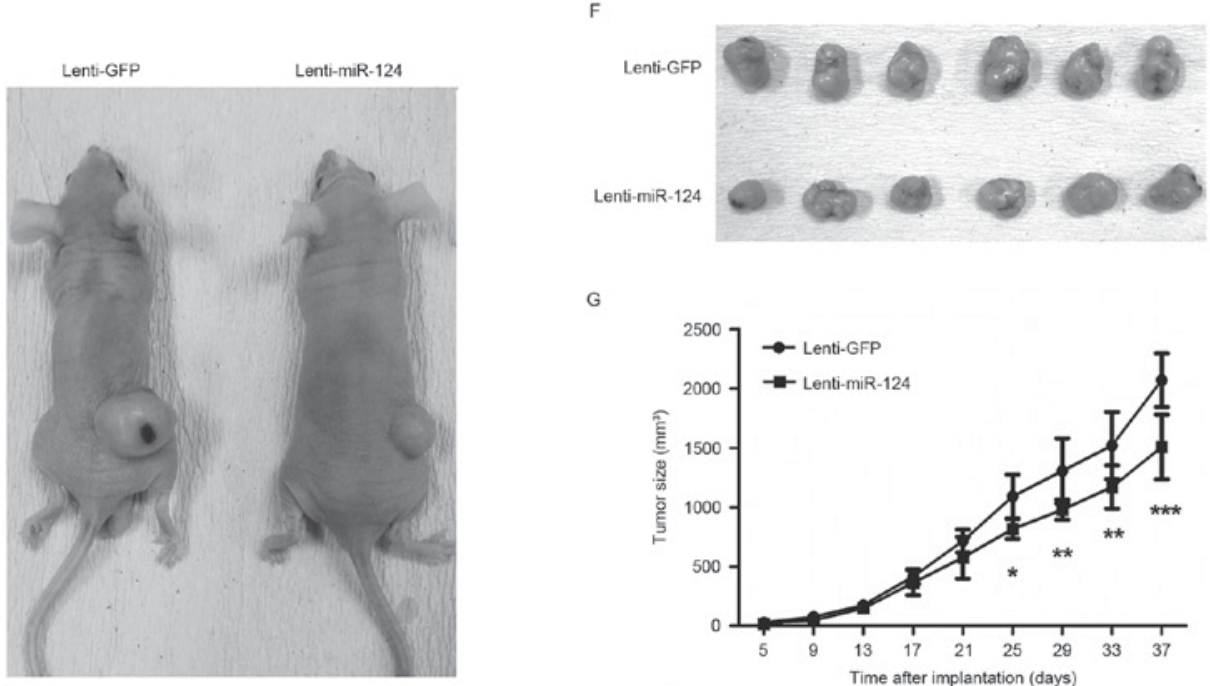

Figure 2. Lentivirus-mediated overexpression of miR-124 suppresses gastric cancer cell growth, migration and invasion. (A and B) Cell Counting kit-8 assays showed that overexpression of miR-124 inhibited SGC-7901 (A) and AGS cells (B) growth. (C and D) Transwell assays revealing that overexpression of miR-124 inhibited SGC-7901 and AGS cells migration and invasion. Micrographs were captured at magnification, $\mathrm{x} 200$. ${ }^{* * *} \mathrm{P}<0.001$ (vs. the negative control group). (E and F) Overexpression of miR-124 in gastric cancer cells inhibited the growth of xenografts in nude mice. SGC-7901 cells transduced with Lenti-miR-124 or Lenti-GFP were injected subcutaneously into the right back to establish xenograft model. At the final time point (37 days after the first injection), tumors in the two groups measured (E) in situ and (F) following resection were shown for the two groups. (G) Statistical analysis of the tumor volume in Lenti-miR-124 and Lenti-GFP groups revealed that overexpression of miR-124 significantly delayed tumor growth compared with the control group. miR-124, microRNA-124; Lenti-miR-124, lentiviral vector expressing miR-124; Lenti-GFP, lentiviral vector expressing miR-124 and green fluorescent protein. ${ }^{*}<0.05$, ${ }^{* *} \mathrm{P}<0.01,{ }^{* * * *} \mathrm{P}<0.001$ 
A

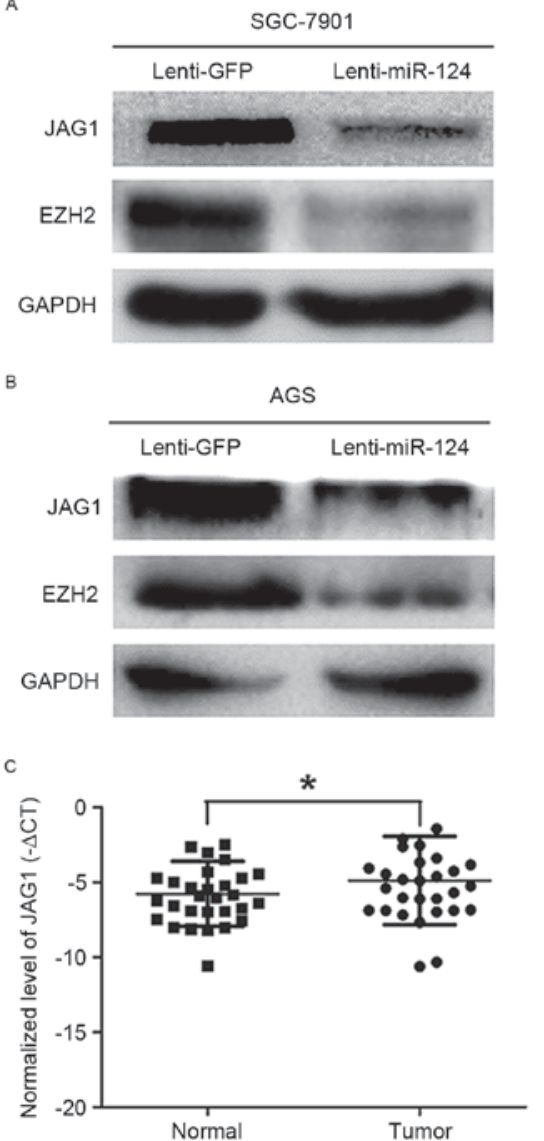

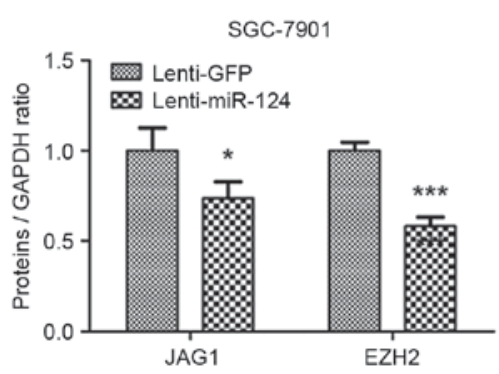

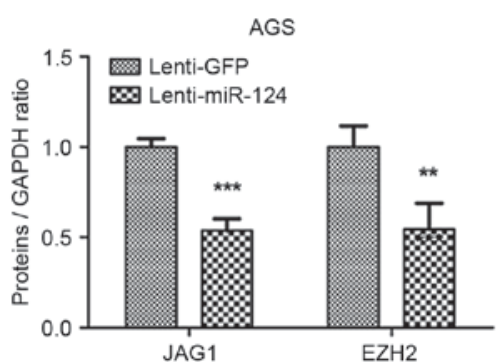

D

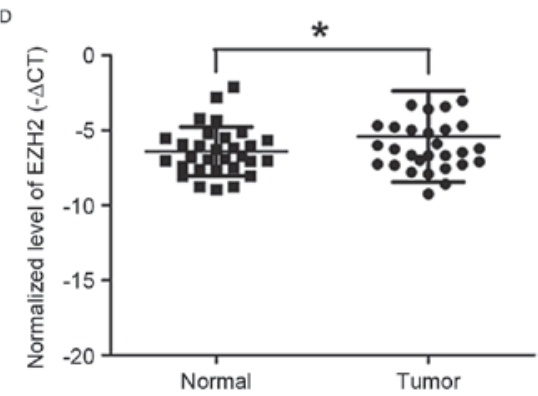

Figure 3. Lentivirus-mediated overexpression of miR-124 repressed the expression of JAG1 and EZH2 in gastric cancer cell lines. (A and B) Western blot analysis revealed the expression levels of JAG1 and EZH2 in gastric cancer SGC-7901 (A) and AGS (B) cells transduced with Lenti-miR-124 or Lenti-GFP. (C) JAG1 mRNA levels were determined in 30 fresh GC tissues and matched adjacent non-tumor tissues using RT-qPCR. Paired t-test was used to analyze the difference between two groups. (D) EZH2 mRNA levels were determined by RT-qPCR in the same cases of fresh GC tissues and paired adjacent non-tumor tissues as previously used to detect JAG1 mRNA levels. Paired t-test was used to analyze the difference between the two groups. miR-124, microRNA-124; Lenti-miR-124, lentiviral vector expressing miR-124; Lenti-GFP, lentiviral vector expressing miR-124 and green fluorescent protein; JAG1, jagged1; EZH2, enhancer of zeste homolog 2 ; GC, gastric cancer; $\mathrm{RT}-\mathrm{qPCR}$, reverse transcription-quantitative polymerase chain reaction. ${ }^{*} \mathrm{P}<0.05,{ }^{* *} \mathrm{P}<0.01,{ }^{* * * *} \mathrm{P}<0.001$.

Overexpression of miR-124 repressed the expression of the JAG1 and EZH2 in GC cell lines. JAG1 and EZH2 have been reported to be targets of miR-124 $(7,10,21)$. JAG1 and EZH2 protein expression levels were downregulated by overexpression of miR-124 in SGC-7901 and AGS GC cell lines (Fig. 3A and B). RT-qPCR was used to quantify the levels of JAG1 and EZH2 in $30 \mathrm{GC}$ and paired normal gastric mucosa tissues, which confirmed the significant upregulation of JAG1 and EZH2 in GC tissues (Fig. 3C and D). These results indicated that the overexpression of miR-124 could repress JAG1 and EZH2 expression in GC cells.

Silencing JAG1 or EZH2 prevented GC cells growth, migration and invasion, and downregulated the expression levels of fibronectin and vimentin. To investigate the function of JAG1 in GC, specific siRNAs were used to silence the expression of JAG1 in SGC-7901 cells (Fig. 4A). The CCK8-based viability assay revealed a significant decrease in the proliferation of SGC-7901 cells following knockdown of JAG1, compared with the NC group (Fig. 4B). Transwell migration and invasion assays revealed a significant decrease in the migratory and invasive ability of GC cells knocked down for JAG1 expression (Fig. 4C). EZH2 was also silenced in SGC-7901 using a lentiviral vector-mediated shRNA against EZH2 (Fig. 4D). As Fig. 4E depicts, suppression of EZH2 led a marked decrease in the GC cell growth. Moreover, knockdown of EZH2 markedly decreased the migration and invasion ability of GC cells (Fig. 4F). Combined with the aforementioned experiments, which demonstrated the antitumor function of miR-124 in GC, these results indicated that miR-124 serves a tumor-suppressive role, at least partly through directly repressing JAG1 and $\mathrm{EZH} 2$ in GC.

Fibronectin and vimentin, the mesenchymal markers, were then detected by western blotting. As shown in Fig. 4G, there was significant decrease in the expression levels of fibronectin and vimentin in GC cells overexpressing miR-124 (Fig. 4G). Knockdown of JAG1 or EZH2 also downregulated fibronectin and vimentin expression in GC cells (Fig. 4G).

\section{Discussion}

miRNAs are small, noncoding RNAs $\sim 22$ nucleotides in length that have notable functions in development, cell differentiation and regulation of the cell cycle and apoptosis (3). miRNAs are dysregulated in various types of cancer, and detection of miRNA levels promises to be a novel diagnostic indicator (22). 

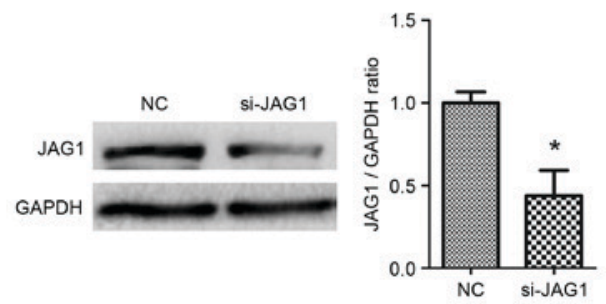

C

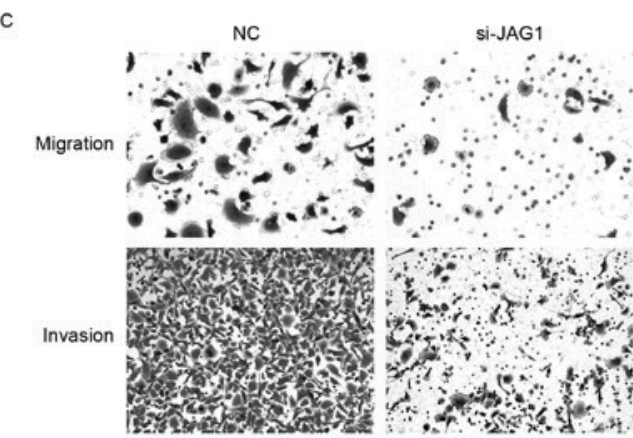

D

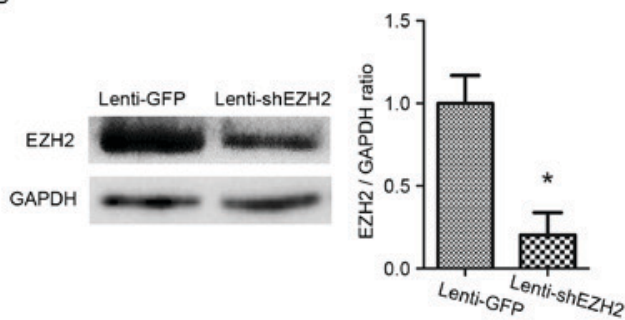

F Lenti-GFP Lenti-shEZH2
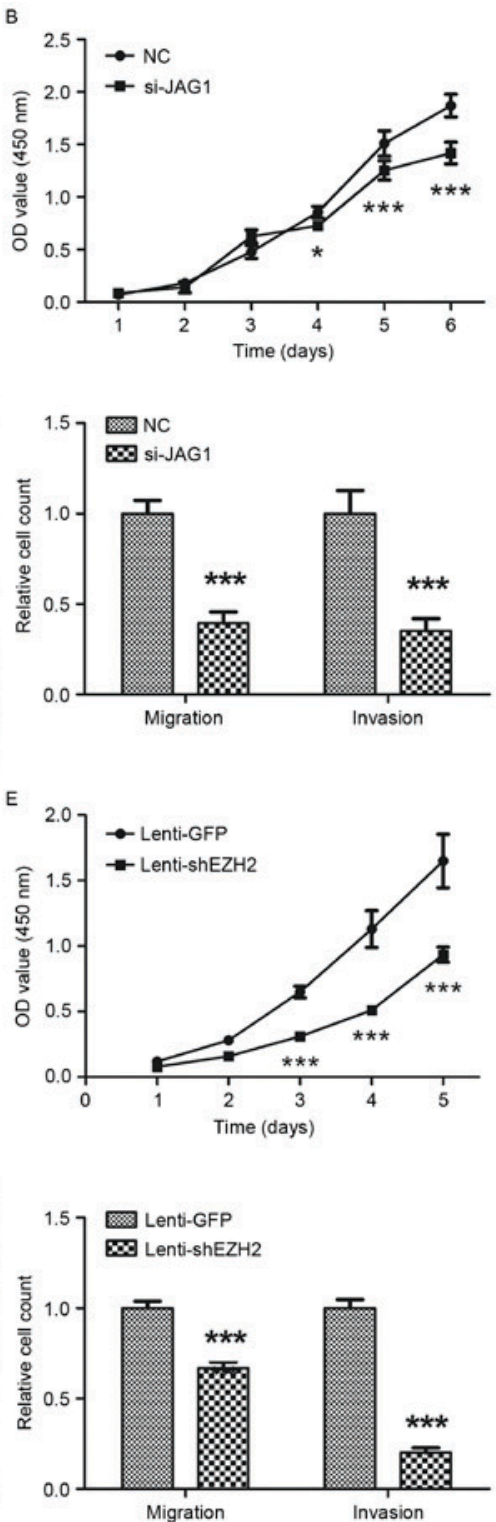
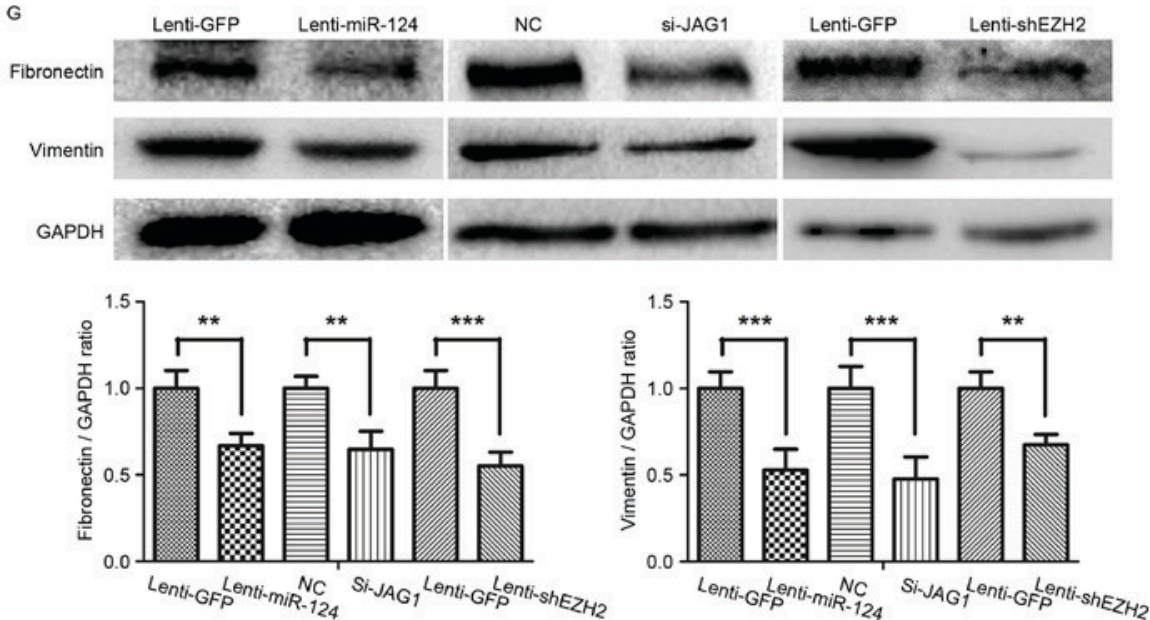

Figure 4. Silencing JAG1 or EZH2 prevents gastric cancer cell growth, migration and invasion. (A) Western blotting revealing the result of JAG1 knockdown with JAG1 siRNA in gastric cancer SGC-7901 cells; (B) CCK8 assays showed that knockdown of JAG1 inhibited growth of SGC-7901 cells; (C) Knockdown of JAG1 inhibited the migration and invasion abilities of SGC-7901 cell (magnification, x200); (D) Western blotting depicting the knockdown of EZH2 by lentiviral vector-mediated shRNA in SGC-7901. (E) CCK8 assays showed that knockdown of EZH2 inhibited cell growth of SGC-7901 cells. (F) The migratory and invasive abilities of gastric cancer cell line SGC-7901 are reduced by transfection with shRNA for EZH2 (magnification, $\mathrm{x} 200$ ). (G) The levels of fibronectin and vimentin protein expression, as determined by western blot analysis. JAG1, jagged1; EZH2, enhancer of zeste homolog 2; siRNA, small interfering RNA; CCK8, Cell Counting kit-8; shRNA, short hairpin RNA. ${ }^{*} \mathrm{P}<0.05,{ }^{* *} \mathrm{P}<0.01,{ }^{* * *} \mathrm{P}<0.001$. 
miRNAs can function as oncogenes or tumor suppressor genes in cancer, aiming at restoration of tumor suppressor genes and silence of oncogenes would be a novel anticancer therapy (23). The inhibitory effect of miR-124 in tumor growth and migration, as well as dysregulation of miR-124 has been reported in various tumor types (6-9). In a previous (13) and the present study, miR-124 was downregulated in GC tissues and cell lines. DNA hypermethylation at $\mathrm{CpG}$ sites is believed to lead to the inactivation of tumor suppressor genes or miRNAs in human cancer (24). To determine whether the methylation of miR-124 occurs predominantly in GC, the methylation status of three $\mathrm{CpG}$ sites of miR-124 gene was evaluated using methylation-specific PCR in 20 GC tissues. The degree of methylation of three $\mathrm{CpG}$ sites of the miR-124 gene in cancer tissues was higher than those in normal gastric mucosa. These data indicated that the deregulation of miR-124 in GC maybe due to the hypermethylation status of miR-124.

Previous studies have revealed that downregulation of miR-124 promotes cancer progression, and forced expression of miR-124 suppresses cancer cell proliferation and inhibits metastasis in specific cancer types including lung, breast and prostate cancer $(7,25-28)$. In the present study, miR-124 was overexpressed using a lentiviral vector system in GC cells; it was found that miR-124 inhibited GC cell growth in vitro and in vivo, and prevented GC cells migration and invasion. These findings indicated that miR-124 may be a potential therapeutic target of GC.

A prior study reported that miR-124 directly targets JAG1(13). It has also been reported that miR-124 directly targets EZH2 by binding the 3'-untranslated region of EZH2 (21). JAG1, as a Notch ligand, was found to be upregulated in GC tissues compared with matched normal tissues in the present study. Notch signaling is a highly conserved cell-signaling pathway that is involved in cell proliferation, differentiation and survival (29). Dysregulation of the Notch signaling pathway often promotes tumor formation and progression. Silencing of JAG1 by siRNA inhibited GC cell growth, migration and invasion. Similar results were found in GC cells transduced with shRNAs targeting EZH2. The present study also determined that EZH2 was upregulated in GC tissues compared with corresponding normal gastric tissues. Silencing EZH2 has also been found to inhibit the proliferation of GC cells and the invasive ability of hepatocellular carcinoma (HCC) cells $(10,21)$. Epithelial-mesenchymal transition is known to be a key event in the invasion and metastasis of cancer cells $(10,30)$. Owing to the effects of miR-124 on GC cell migration and invasion, fibronectin and vimentin, as the mesenchymal markers, were detected in GC cells transduced with Lenti-miR-124. The expression of fibronectin and vimentin were downregulated by miR-124 in GC cells. Similarly, knockdown of JAG1 and EZH2 suppressed fibronectin and vimentin expression. These results provide evidence that the antitumor ability of miR-124 could be partly due to targeting of JAG1 and EZH2 expression. However, further study is required to confirm this conclusion.

In summary, the results of the present study revealed that miR-124 genes were frequently methylated and the expression of miR-124 was downregulated in GC tissues. The lentivirus-mediated overexpression of miR-124 suppressed
GC cell growth, migration and invasion, and downregulated the expressions of fibronectin and vimentin through repression of JAG1 and EZH2. These findings indicate that the lentivirus-mediated overexpression of miR-124 may be a potential therapeutic stategy against GC.

\section{Acknowledgements}

Not applicable.

\section{Funding}

The present study was supported by grants from the National Natural Science Foundation of China (no. 81672385), the Science Research Foundation of National Health and Family Planning commission of China (no. WKJ-ZJ-1416) and Zhejiang Provincial Natural Science Foundation of China (no. LY14H16004).

\section{Availability of data and materials}

All data generated or analyzed during this study are included in this published article.

\section{Authors' contributions}

YP, AW, FX, and CC conducted the study, performed the statistical analysis. YP, LJ and RJ performed the study design and drafted the manuscript. All the authors participated in the discussion have read and approved the final manuscript.

\section{Ethics approval and consent to participate}

The collection and analysis of clinical specimens were approved by the Institutional Review Board of the First Affiliated Hospital of Wenzhou Medical University. Written informed consent was obtained from the patients prior to participation.

\section{Consent for publication}

Not applicable.

\section{Competing interests}

The authors declare that they have no competing interests.

\section{References}

1. Torre LA, Bray F, Siegel RL, Ferlay J, Lortet-Tieulent J and Jemal A: Global cancer statistics, 2012. CA Cancer J Clin 65: 87-108, 2015

2. Lin M, Shi C, Lin X, Pan J, Shen S, Xu Z and Chen Q: sMicroRNA-1290 inhibits cells proliferation and migration by targeting FOXA1 in gastric cancer cells. Gene 582: 137-142, 2016.

3. Garzon R, Calin GA and Croce CM: MicroRNAs in Cancer. Annu Rev Med 60: 167-179, 2009.

4. Hayes EL and Lewis-Wambi JS: Mechanisms of endocrine resistance in breast cancer: An overview of the proposed roles of noncoding RNA. Breast Cancer Res 17: 40, 2015.

5. Sun Y, Luo ZM, Guo XM, Su DF and Liu X: An updated role of microRNA-124 in central nervous system disorders: A review. Front Cell Neurosci 9: 193, 2015. 
6. Feng T, Shao F, Wu Q, Zhang X, Xu D, Qian K, Xie Y, Wang S, $\mathrm{Xu}$ N, Wang Y and Qi C: miR-124 downregulation leads to breast cancer progression via LncRNA-MALAT1 regulation and CDK4/E2F1 signal activation. Oncotarget 7: 16205-16216, 2016.

7. Shi XB, Ma AH, Xue L, Li M, Nguyen HG, Yang JC, Tepper CG Gandour-Edwards R, Evans CP, Kung HJ, et al: miR-124 and androgen receptor signaling inhibitors repress prostate cancer growth by downregulating androgen receptor splice variants, EZH2 and Src. Cancer Res 75: 5309-5317, 2015.

8. Zu L, Xue Y, Wang J, Fu Y, Wang X, Xiao G, Hao M, Sun X, Wang Y, Fu G and Wang J: The feedback loop between miR-124 and TGF- $\beta$ pathway plays a significant role in non-small cell lung cancer metastasis. Carcinogenesis 37: 333-343, 2016.

9. Chen Z, Liu S, Tian L, Wu M, Ai F, Tang W, Zhao L, Ding J, Zhang L and Tang A: miR-124 and miR-506 inhibit colorectal cancer progression by targeting DNMT3B and DNMT1. Oncotarget 6: 38139-38150, 2015.

10. Xie L, Zhang Z, Tan Z, He R, Zeng X, Xie Y, Li S, Tang G, Tang $\mathrm{H}$ and $\mathrm{He} \mathrm{X}$ : MicroRNA-124 inhibits proliferation and induces apoptosis by directly repressing $\mathrm{EZH} 2$ in gastric cancer. Mol Cell Biochem 392: 153-159, 2014.

11. Xia J, Wu Z, Yu C, He W, Zheng H, He Y, Jian W, Chen L, Zhang L and $\mathrm{Li} \mathrm{W}$ : miR-124 inhibits cell proliferation in gastric cancer through down-regulation of SPHK1. J Pathol 227: 470-480, 2012.

12. Hu CB, Li QL, Hu JF, Zhang Q, Xie JP and Deng L: miR-124 inhibits growth and invasion of gastric cancer by targeting ROCK1. Asian Pac J Cancer Prev 15: 6543-6546, 2014.

13. Jiang L, Lin T, Xu C, Hu S, Pan Y and Jin R: miR-124 interacts with the Notch1 signalling pathway and has therapeutic potential against gastric cancer. J Cell Mol Med 20: 313-322, 2016.

14. Liu L, Ye JX, Qin YZ, Chen QH and Ge LY: Evaluation of miR-29c, miR-124, miR-135a and miR-148a in predicting lymph node metastasis and tumor stage of gastric cancer. Int J Clin Exp Med 8: 22227-22236, 2015.

15. Jiang L, Lai YK, Zhang J, Wang H, Lin MC, He ML and Kung HF: Targeting S100P inhibits colon cancer growth and metastasis by Lentivirus-mediated RNA interference and proteomic analysis. Mol Med 17: 709-716, 2011.

16. Chen Y, Lin MC, Wang H, Chan CY, Jiang L, Ngai SM, Yu J, He ML, Shaw PC, Yew DT, et al: Proteomic analysis of EZH2 downstream target proteins in hepatocellular carcinoma. Proteomics 7: 3097-3104, 2007.

17. Steg AD, Katre AA, Goodman B, Han HD, Nick AM, Stone RL, Coleman RL, Alvarez RD, Lopez-Berestein G, Sood AK and Landen CN: Targeting the notch ligand JAGGED1 in both tumor cells and stroma in ovarian cancer. Clin Cancer Res 17: 5674-5685, 2011.
18. Livak KJ and Schmittgen TD: Analysis of relative gene expression data using real-time quantitative PCR and the 2(-Delta Delta C(T)) method. Methods 25: 402-408, 2001.

19. Wang $\mathrm{P}$, Chen L, Zhang J, Chen H, Fan J, Wang K, Luo J, Chen Z, Meng Z and Liu L: Methylation-mediated silencing of the miR-124 genes facilitates pancreatic cancer progression and metastasis by targeting Rac1. Oncogene 33: 514-524, 2014

20. Furuta M, Kozaki KI, Tanaka S, Arii S, Imoto I and Inazawa J: miR-124 and miR-203 are epigenetically silenced tumor-suppressive microRNAs in hepatocellular carcinoma. Carcinogenesis 31: 766-776, 2010.

21. Zheng F, Liao YJ, Cai MY, Liu YH, Liu TH, Chen SP, Bian XW, Guan XY, Lin MC, Zeng YX, et al: The putative tumour suppressor microRNA-124 modulates hepatocellular carcinoma cell aggressiveness by repressing ROCK2 and EZH2. Gut 61: 278-289, 2012.

22. Iorio MV and Croce CM: MicroRNA dysregulation in cancer: Diagnostics, monitoring and therapeutics. A comprehensive review. EMBO Mol Med 4: 143-159, 2012.

23. Rupaimoole R, Calin GA, Lopez-Berestein G and Sood AK: miRNA deregulation in cancer cells and the tumor microenvironment. Cancer Discov 6: 235-246, 2016.

24. Saito Y: Alterations of epigenetics and microRNAs in cancer and cancer stem cell. Front Genet 5: 283, 2014.

25. Zhang N, Huang Y, Wu F, Zhao Y, Li X, Shen P, Yang L, Luo Y, Yang L and He G: Codelivery of a miR-124 mimic and obatoclax by cholesterol-penetratin micelles simultaneously induces apoptosis and inhibits autophagic flux in breast cancer in vitro and in vivo. Mol Phar 13: 2466-2483, 2016.

26. Feng T, Xu D, Tu C, Li W, Ning Y, Ding J, Wang S, Yuan L, Xu N, Qian K, et al: MiR-124 inhibits cell proliferation in breast cancer through downregulation of CDK4. Tumour Biol 36: 5987-5997, 2015.

27. Zhang W, Mao YQ, Wang H, Yin WJ, Zhu SX and Wang WC: MiR-124 suppresses cell motility and adhesion by targeting talin 1 in prostate cancer cells. Cancer Cell Int 15: 49, 2015.

28. Sun Y, Ai X, Shen S and Lu S: NF- $\kappa$ B-mediated miR-124 suppresses metastasis of non-small-cell lung cancer by targeting MYO10. Oncotarget 6: 8244-8254, 2015.

29. Yuan X, Wu H, Xu H, Xiong H, Chu Q, Yu S, Wu GS and Wu K: Notch signaling: An emerging therapeutic target for cancer treatment. Cancer Lett 369: 20-27, 2015.

30. Thiery JP: Epithelial-mesenchymal transitions in tumour progression. Nat Rev Cancer 2: 442-454, 2002. 